EDITORIAL

\title{
Early Detection for Maximizing Stroke Prevention
}

Can J Neurol Sci. 2013; 40: 135-136

'Living at risk is jumping off the cliff and building your wings on the way down'.

Ray Bradbury (1920 - 2012), science fiction writer

In the last two decades, we were exposed to two major 'epidemiological tsunamis': the dramatic increase in the prevalence of obesity and diabetes, especially in children and young adults. ${ }^{1}$ This has also been associated with an increase in the prevalence of cardiovascular and cerebrovascular disease at a young age $e^{2,3}$, likely related with different pathways resulting in premature and advanced atherosclerosis. As known, advanced atherosclerosis is a risk factor for both intra- and extra-cranial large (and small) artery diseases. ${ }^{4}$

In the present study ${ }^{5}$, Dr. Lam and colleagues evaluated 339 patients with asymptomatic carotid stenosis (ACS) greater than $60 \%$ by doppler ultrasound. Participants underwent transcranial doppler for detection of intracranial stenosis and microemboli. They were followed up during three years. The main outcome was time to stroke, transient ischemic attack (TIA) or death. Intracranial stenosis was present in $112(33 \%)$ patients with ACS; $17 \%$ had diabetes. The authors found an incident risk of stroke, TIA or death of $38 \%$ among patients with microemboli compared to $10 \%$ for those without microemboli $(\mathrm{p}=0.0001)$. Similar findings were observed for patients with intracranial stenosis $(18 \%$ vs $10 \%$; $=0.042)$. Furthermore, patients with diabetes were significantly more likely to have intracranial stenosis $(45 \%$ vs. $29 \%, p=0.014)$, microemboli $(38 \%$ vs. $10 \%$, $\mathrm{p}<0.0001)$, and had a higher incident risk of stroke, TIA or death ( $21 \%$ vs. $11 \%$; $=0.024)$. These findings were confirmed in the survival analysis.

The diagnosis and management of asymptomatic carotid stenosis has changed in the last 10 or 15 years. Asymptomatic carotid stenosis is prevalent, affecting $2 \%$ to $8 \%$ of the general population. ${ }^{6}$ Considering the population aging, the rising of obesity and diabetes, and the increased risk of atherosclerosis with age, clinicians are likely to provide care to more complex patients with ACS. ${ }^{6,7}$

Previous randomized trials in the 1980's showed a moderate reduction in the risk of stroke for carotid endarterectomy (CEA) compared to medical management when the perioperative risk was below 3\% ${ }^{6,8,9}$ Despite a different outcome measure, both the Asymptomatic Carotid Stenosis trial (ACAS) and ACST-trial showed similar results: a small absolute risk reduction of 5-6\% for CEA vs. medical treatment. ${ }^{8,9}$ However, these trials ${ }^{8,9}$ were conducted before the development of statins, angiotensin converting enzyme inhibitors, and new antiplatelet agents. As a result, the recent Guidelines on the Management of Patients with Extracranial Carotid and Vertebral Artery Disease questioned the benefit of carotid revascularization for patients with ACS as the number needed to treat and to prevent an ipsilateral disabling or fatal stroke is greater than 70, and probably higher depending on the studied outcome. ${ }^{4}$ Carotid stenting has not proven better than current medical therapy alone for ACS (Class IIb; Level of Evidence B). ${ }^{6}$ Furthermore, the recent results of the Stenting and Aggressive Medical Management for Preventing Recurrent stroke in Intracranial Stenosis (SAMPPRIS) trial also reinforce the importance of aggressive medical therapy in patients with intracranial arterial stenosis. The probability of the primary end point (stroke or death at 30-days) was reduced by $60 \%$ in the medical treatment arm (14.7\% angioplasty/stent vs $5.8 \%$ in the medical-management group; $\mathrm{p}=0.002)$.

\section{Lessons learned and limitations}

Dr. Lam and colleagues ${ }^{5}$ acknowledged some limitations. More importantly, we should bear in mind this is a small, single centre study in a very high risk population. For example, $22 \%$ of participants had history of TIA before enrolment in the study, $22 \%$ had experienced a myocardial infarction, $16 \%$ had carotid occlusion on one side, and a higher prevalence of elevated homocysteine, all markers of atherosclerosis. Unfortunately, ethnicity was not available. Interestingly, the same group showed in a previous publication that microemboli were present in $12.6 \%$ before 2003 and $3.7 \%$ since 2003 (p<0.001). ${ }^{10}$ The decline in microemboli coincided with better control of plasma lipids, a $66 \%$ reduction in the progression of carotid plaque area and significantly lower vascular events $(17.6 \%$ vs. $5.6 \%$ after $2003 ; \mathrm{p}<0.001)$ over two years. Of note, these achievements were likely due to the intensive medical therapy as shown by the increased use (before and after 2003) of statin (13.6\% to $33.8 \%$; $\mathrm{p}<0.001)$, antihypertensive $(44.8 \%$ to $58.3 \% ; \mathrm{p}=0.02)$ and antiplatelet ( $81.4 \%$ to $98.2 \%$ ) agents. ${ }^{10}$ Knowing the intensive therapeutic program and comprehensive approach at the Stroke Prevention \& Atherosclerosis Research Centre (SPARC) where the study was conducted, the remaining unknowns relate to current participants' adherence and compliance with the prescribed stroke prevention therapy. We anticipate the Canadian Fabry Stroke Screening Initiative (CFSSI), an ongoing prospective multicenter cohort study of young adults with cryptogenic stroke across Canada, will also reveal useful information on premature atherosclerosis. ${ }^{11}$

In summary, the present study from Dr. Lam et al provides novel information on subgroups with ACS at higher risk of cardio- and cerebro-vascular events. Vascular imaging may help stratify patients. Aggressive medical management in conjunction with lifestyle modification and exercising are proven and effective strategies to decrease that risk.

Gustavo Saposnik University of Toronto and the Institute for Clinical Evaluative Sciences \& Li Ka Shing Knowledge Institute Toronto, Ontario, Canada 


\section{SOURCES OF FUNDING}

Dr. Saposnik is supported by the Distinguished Clinician Scientist Award from Heart and Stroke Foundation of Canada.

\section{REFERENCES}

1. Roger VL, Go AS, Lloyd-Jones DM, et al. Heart disease and stroke statistics--2012 update: a report from the American Heart Association. Circulation. 2012;125:e2-220.

2. Kissela BM, Khoury JC, Alwell K, et al. Age at stroke: temporal trends in stroke incidence in a large, biracial population. Neurology. 2012;79:1781-7.

3. Lee LK, Bateman BT, Wang S, Schumacher HC, Pile-Spellman J, Saposnik G. Trends in the hospitalization of ischemic stroke in the United States, 1998-2007. Int J Stroke. 2012;7:195-201.

4. Brott TG, Halperin JL, Abbara S, et al. 2011 ASA/ACCF/AHA/ AANN/AANS/ACR/ASNR/CNS/SAIP/SCAI/SIR/SNIS/SVM/ SVS guideline on the management of patients with extracranial carotid and vertebral artery disease: executive summary. A report of the American College of Cardiology Foundation/American Heart Association Task Force on Practice Guidelines, and the American Stroke Association, American Association of Neuroscience Nurses, American Association of Neurological Surgeons, American College of Radiology, American Society of Neuroradiology, Congress of Neurological Surgeons, Society of Atherosclerosis Imaging and Prevention, Society for Cardiovascular Angiography and Interventions, Society of Interventional Radiology, Society of Neurointerventional Surgery, Society for Vascular Medicine, and Society for Vascular Surgery. Circulation. 2011;124:489-532.
5. Lam TD, Lammers S, Munoz C, Tamayo A, Spence JD. Diabetes, intracranial stenosis and microemboli in asymptomatic carotid stenosis. Can J Neurol Sci. 2013;40(2):177-181.

6. Goldstein LB, Bushnell CD, Adams RJ, et al. Guidelines for the primary prevention of stroke: a guideline for healthcare professionals from the American Heart Association/American Stroke Association. Stroke. 2011;42:517-84.

7. Saposnik G, Cote R, Phillips S, et al. Stroke outcome in those over 80: a multicenter cohort study across Canada. Stroke. 2008;39: 2310-17.

8. Endarterectomy for asymptomatic carotid artery stenosis. Executive Committee for the Asymptomatic Carotid Atherosclerosis Study. JAMA. 1995;273:1421-8.

9. Halliday A, Mansfield A, Marro J, et al. Prevention of disabling and fatal strokes by successful carotid endarterectomy in patients without recent neurological symptoms: randomised controlled trial. Lancet. 2004;363:1491-502.

10. Spence JD, Coates V, Li H, et al. Effects of intensive medical therapy on microemboli and cardiovascular risk in asymptomatic carotid stenosis. Arch Neurol. 2010;67:180-6.

11. Saposnik G, Lanthier S, Mamdani M, et al. Fabry's disease: a prospective multicenter cohort study in young adults with cryptogenic stroke. Int J Stroke. 2012;7:265-73. 